\title{
Uniqueness and existence of positive solutions for the fractional integro-differential equation
}

Ying Wang ${ }^{1 *}$ and Lishan Liu ${ }^{2,3}$

${ }^{*}$ Correspondence:
lywy1981@163.com
'School of Science, Linyi University,
Linyi, Shandong 276000, People's
Republic of China
Full list of author information is
available at the end of the article

available at the end of the article

\begin{abstract}
In this paper, we study the uniqueness and existence of positive solutions for the fractional integro-differential equation with the integral boundary value problem. By means of the Banach contraction principle and the Krasnoselskii fixed point theorem, the sufficient conditions on the uniqueness and existence of positive solutions are investigated. An example is given to illustrate the main results.
\end{abstract}

MSC: $34 \mathrm{~A} 08 ; 34 \mathrm{~B} 18$

Keywords: fractional integro-differential equation; integral conditions; uniqueness; existence

\section{Introduction}

It is widely recognized that the memory and hereditary properties of various materials and processes are well predicted by using fractional differential operators. The differential equation with fractional order derivative has recently proven to be a strong tool in the modeling of many phenomena in various fields of science and engineering $[1,2]$. The fractional differential equation has made a profound impact on some areas such as viscoelasticity, diffusion procedures, relaxation vibrations, electrochemistry, signal and image processing, mechatronics, physics, and control theory; see [3-5].

The Volterra model for population growth of a species within a closed system is characterized by a nonlinear fractional integro-differential equation in the following form:

$$
{ }^{c} D^{\alpha} p(t)=a p(t)-b p^{2}(t)-c p(t) \int_{0}^{t} p(x) d x, \quad p(0)=p_{0},
$$

where ${ }^{c} D^{\alpha}$ denotes the Caputo fractional derivative of order $0<\alpha \leq 1, p(t)$ is the scaled population of identical individuals, $t$ denotes the time, $a>0$ is the birth rate coefficient, $b>0$ is the crowding coefficient, and $c>0$ is the toxicity coefficient, which denotes the essential behavior of the population evolution before its level falls to zero in the long run [6, 7]. Besides, the nonlinear oscillation of earthquake can be modeled with fractional derivatives [8], and the fluid-dynamic traffic model with fractional derivatives can eliminate the deficiency arising from the assumption of continuum traffic flow [9]. In the characteriza-

(c) The Author(s) 2017. This article is distributed under the terms of the Creative Commons Attribution 4.0 International License (http://creativecommons.org/licenses/by/4.0/), which permits unrestricted use, distribution, and reproduction in any medium, provided you give appropriate credit to the original author(s) and the source, provide a link to the Creative Commons license, and indicate if changes were made. 
tion of viscoelasticity's Hook law, the five parameter generalized Zener model

$$
x(t)+a D_{t}^{\alpha} x(t)=b y(t)+c D_{t}^{\beta} y(t)
$$

or the three parameter generalized Maxwell model

$$
x(t)+a D_{t}^{\alpha} y(t)=b z(t)
$$

are often used. With suitable initial or boundary conditions, the existence and nonexistence of positive solutions for the above equations are significant and serviceable.

Since theoretical results can help to get an in-depth understanding for the fractional order model, motivated by the mentioned equation models and their application background, in this paper, we concentrate on the more complicated and abstract fractional boundary value problem (FBVP):

$$
\left\{\begin{array}{l}
{ }^{c} D^{\alpha} u(t)+f(t, u(t), T u(t), S u(t))=0, \quad 0<t<1, \\
u(0)=b_{0}, \quad u^{\prime}(0)=b_{1}, \quad \ldots, \quad u^{(n-3)}(0)=b_{n-3}, \\
u^{(n-1)}(0)=b_{n-1}, \quad u(1)=\mu \int_{0}^{1} u(s) d s,
\end{array}\right.
$$

where $n-1<\alpha \leq n, 0 \leq \mu<n-1, n \geq 3, b_{i} \geq 0(i=1,2, \ldots, n-3, n-1),{ }^{c} D^{\alpha}$ is the Caputo fractional derivative. $f: J \times \mathbb{R}_{+} \times \mathbb{R}_{+} \times \mathbb{R}_{+} \rightarrow \mathbb{R}_{+}$is a given function satisfying some assumptions that will be specified later, $J=[0,1], \mathbb{R}_{+}=[0,+\infty) . T, S$ are given by

$$
(T x)(t)=\int_{0}^{t} K(t, s) x(s) d s, \quad(S x)(t)=\int_{0}^{1} H(t, s) x(s) d s
$$

with $k^{*}=\sup _{t \in J} \int_{0}^{t} K(t, s) d s, h^{*}=\sup _{t \in J} \int_{0}^{1} H(t, s) d s$, in which $K \in C\left(D, \mathbb{R}_{+}\right), D=\{(t, s) \in$ $J \times J: t \geq s\}, H \in C\left(J \times J, \mathbb{R}_{+}\right)$.

For the past few decades, many researchers have tried to model real processes using the fractional calculus. In the mathematical context, several interesting results about the existence of positive solutions for fractional equation models have been reported [10-25]. In [10], the following boundary value problem of the fractional differential equation was considered:

$$
\left\{\begin{array}{l}
{ }^{c} D^{\alpha} u(t)+f(t, u(t))=0, \quad 0<t<1 \\
u(0)=u^{\prime}(1)=u^{\prime \prime}(0)=0
\end{array}\right.
$$

where $2<\alpha \leq 3,{ }^{c} D^{\alpha}$ is the Caputo fractional derivative. $f:(0,1] \times \mathbb{R}_{+} \rightarrow \mathbb{R}_{+}$is continuous. By the Guo-Krasnoselskii fixed point theorem and the nonlinear alternative of LeraySchauder type, the authors in [10] established the existence of positive solutions to the problem (1.2).

Cabada and Wang [14] demonstrated some existence results for positive solutions to the FBVP (1.3) relying on the known Guo-Krasnoselskii fixed point theorem,

$$
\left\{\begin{array}{l}
{ }^{c} D^{\alpha} u(t)+f(t, u(t))=0, \quad 0<t<1, \\
u(0)=u^{\prime \prime}(0)=0, \quad u(1)=\lambda \int_{0}^{1} u(s) d s,
\end{array}\right.
$$


where $2<\alpha<3,0<\lambda<2,{ }^{c} D^{\alpha}$ is the Caputo fractional derivative. $f: J \times \mathbb{R}_{+} \rightarrow \mathbb{R}_{+}$is a continuous function. Xu and He [17] researched the FBVP (1.3) for $3<\alpha<4$.

But up to now, when $f$ contains integral operators $T$ and $S$, a fractional differential equation like FBVP (1.1) has seldom been considered, furthermore, in FBVP (1.1), $b_{i} \geq 0$, we generalized the boundary conditions in $[10,14,17]$. Inspired greatly by the above mentioned works, we establish some new existence criteria of uniqueness and existence of positive solutions for FBVP (1.1).

\section{Preliminaries and lemmas}

Denote $C\left(J, \mathbb{R}_{+}\right)$the Banach space of all continuous functions from $J$ into $\mathbb{R}_{+}$with the norm $\|u\|:=\sup \{|u(t)|: t \in J\}, L^{p}\left(J, \mathbb{R}_{+}\right)$the Banach space of all Lebesgue measurable functions from $J$ into $\mathbb{R}_{+}$with the norm $\|y\|_{L^{p}}:=\left(\int_{0}^{1}(y(t))^{p} d t\right)^{\frac{1}{p}}<+\infty, 1 \leq p<+\infty$, respectively. Next, we introduce some basic definitions and properties of the fractional calculus theory and auxiliary lemmas in order to obtain the uniqueness and existence of positive solutions for FBVP (1.1).

Definition 2.1 ([26, 27]) The Caputo fractional order derivative of order $\alpha>0, n-1<\alpha<$ $n, n \in \mathbb{N}$ is defined as

$$
{ }^{c} D^{\alpha} u(t)=\frac{1}{\Gamma(n-\alpha)} \int_{0}^{t}(t-s)^{n-\alpha-1} u^{(n)}(s) d s,
$$

where $u \in C^{n}(J, \mathbb{R}), \mathbb{R}=(-\infty,+\infty), \mathbb{N}$ denotes the natural number set, $n=[\alpha]+1$, and $[\alpha]$ denotes the integer part of $\alpha$.

Definition $2.2([26,27])$ The Riemann-Liouville fractional derivative of order $\alpha>0, n-$ $1<\alpha<n, n \in \mathbb{N}$ is defined as

$$
D^{\alpha} u(t)=\frac{1}{\Gamma(n-\alpha)}\left(\frac{d}{d t}\right)^{n} \int_{0}^{t}(t-s)^{n-\alpha-1} u(s) d s,
$$

where the function $u(t)$ is $n$ times continuously differentiable on $J$.

Definition $2.3([26,27])$ Let $\alpha>0$ and let $u$ be piecewise continuous on $(0,+\infty)$ and integrable on any finite subinterval of $J$. Then, for $t>0$, we call

$$
I^{\alpha} u(t)=\frac{1}{\Gamma(\alpha)} \int_{0}^{t}(t-s)^{\alpha-1} u(s) d s
$$

the Riemann-Liouville fractional integral of $u$ of order $\alpha$.

Lemma 2.1 ([26,27]) Let $n-1<\alpha \leq n$. Then the differential equation ${ }^{c} D^{\alpha} u(t)=0$ has one solution as follows:

$$
u(t)=c_{0}+c_{1} t+c_{2} t^{2}+\cdots+c_{n-1} t^{n-1}
$$

where $c_{i} \in \mathbb{R}(i=1,2, \ldots, n-1), n$ is the smallest integer greater than or equal to $\alpha$. 
Lemma $2.2([26,27])$ Let $n-1<\alpha \leq n, u \in C^{n}[0,1]$. Then

$$
I^{\alpha}\left({ }^{c} D^{\alpha} u\right)(t)=u(t)+c_{0}+c_{1} t+c_{2} t^{2}+\cdots+c_{n-1} t^{n-1},
$$

where $c_{i} \in \mathbb{R}(i=1,2, \ldots, n-1), n$ is the smallest integer greater than or equal to $\alpha$.

For $h \in C(0,1) \cap L^{1}(0,1)$, consider the following FBVP (2.1) with integral boundary value condition:

$$
\left\{\begin{array}{l}
{ }^{c} D^{\alpha} u(t)+h(t)=0, \quad 0<t<1, n-1<\alpha \leq n, \\
u(0)=b_{0}, \quad u^{\prime}(0)=b_{1}, \quad \ldots, \quad u^{(n-3)}(0)=b_{n-3}, \\
u^{(n-1)}(0)=b_{n-1}, \quad u(1)=\mu \int_{0}^{1} u(s) d s
\end{array}\right.
$$

where $n-1<\alpha \leq n, 0 \leq \mu<n-1, n \geq 3, b_{i} \geq 0(i=1,2, \ldots, n-3, n-1)$.

Lemma 2.3 Let $h \in C(0,1) \cap L^{1}(0,1)$, then $F B V P(2.1)$ has an integral representation

$$
\begin{aligned}
u(t)= & -\int_{0}^{t} \frac{(t-s)^{\alpha-1}}{\Gamma(\alpha)} h(s) d s+M(t)+\frac{(n-1) t^{n-2}}{n-1-\mu} \int_{0}^{1} \frac{(1-s)^{\alpha-1}}{\Gamma(\alpha)} h(s) d s \\
& -\frac{\mu(n-1) t^{n-2}}{n-1-\mu} \int_{0}^{1} \frac{(1-s)^{\alpha}}{\alpha \Gamma(\alpha)} h(s) d s+\frac{(\mu \mathcal{M}-M(1))(n-1) t^{n-2}}{n-1-\mu},
\end{aligned}
$$

where $M(t)=b_{0}+b_{1} t+\cdots+\frac{b_{n-3} t^{n-3}}{(n-3) !}+\frac{b_{n-1} t^{n-1}}{(n-1) !}, M(1)=b_{0}+b_{1}+\cdots+\frac{b_{n-3}}{(n-3) !}+\frac{b_{n-1}}{(n-1) !}, \mathcal{M}=$ $b_{0}+\frac{b_{1}}{2}+\cdots+\frac{b_{n-3}}{(n-2) !}+\frac{b_{n-1}}{n !}$.

Proof FBVP (2.1) is equivalent to the following integral equation:

$$
\begin{aligned}
u(t) & =-I^{\alpha} h(t)+\sum_{j=0}^{n-1} \frac{u^{j}(0)}{j !} t^{j} \\
& =-\int_{0}^{t} \frac{(t-s)^{\alpha-1}}{\Gamma(\alpha)} h(s) d s+u(0)+u^{\prime}(0) t+\cdots+\frac{u^{(n-1)}(0) t^{n-1}}{(n-1) !} .
\end{aligned}
$$

Since $u(0)=b_{0}, u^{\prime}(0)=b_{1}, \ldots, u^{(n-3)}(0)=b_{n-3}, u^{(n-1)}(0)=b_{n-1}$, we can deduce that

$$
\begin{aligned}
u(t)= & -\int_{0}^{t} \frac{(t-s)^{\alpha-1}}{\Gamma(\alpha)} h(s) d s \\
& +b_{0}+b_{1} t+\cdots+\frac{b_{n-3} t^{n-3}}{(n-3) !}+\frac{u^{(n-2)}(0) t^{n-2}}{(n-2) !}+\frac{b_{n-1} t^{n-1}}{(n-1) !} .
\end{aligned}
$$

The other boundary condition $u(1)=\mu \int_{0}^{1} u(s) d(s)$ implies that

$$
\begin{aligned}
\frac{u^{(n-2)}(0)}{(n-2) !}= & \mu \int_{0}^{1} u(s) d s+\int_{0}^{1} \frac{(1-s)^{\alpha-1}}{\Gamma(\alpha)} h(s) d s \\
& -b_{0}-b_{1}-\cdots-\frac{b_{n-3}}{(n-3) !}-\frac{b_{n-1}}{(n-1) !} .
\end{aligned}
$$


Hence, substituting (2.5) into (2.4), we have

$$
\begin{aligned}
u(t)= & -\int_{0}^{t} \frac{(t-s)^{\alpha-1}}{\Gamma(\alpha)} h(s) d s+b_{0}+b_{1} t+\cdots+\frac{b_{n-3} t^{n-3}}{(n-3) !}+\frac{b_{n-1} t^{n-1}}{(n-1) !} \\
& +t^{n-2}\left(\mu \int_{0}^{1} u(s) d s+\int_{0}^{1} \frac{(1-s)^{\alpha-1}}{\Gamma(\alpha)} h(s) d s-b_{0}-b_{1}\right. \\
& \left.-\cdots-\frac{b_{n-3}}{(n-3) !}-\frac{b_{n-1}}{(n-1) !}\right) .
\end{aligned}
$$

Now, integrate (2.6) from 0 to 1 in both sides, we get

$$
\begin{aligned}
\int_{0}^{1} u(t) d t= & \frac{n-1}{n-1-\mu}\left(-\int_{0}^{1} \frac{(1-s)^{\alpha}}{\alpha \Gamma(\alpha)} h(s) d s+b_{0}+\frac{b_{1}}{2}+\cdots+\frac{b_{n-3}}{(n-2) !}+\frac{b_{n-1}}{n !}\right) \\
& +\frac{1}{n-1-\mu}\left(\int_{0}^{1} \frac{(1-s)^{\alpha-1}}{\Gamma(\alpha)} h(s) d s-b_{0}-b_{1}\right. \\
& \left.-\cdots-\frac{b_{n-3}}{(n-3) !}-\frac{b_{n-1}}{(n-1) !}\right) .
\end{aligned}
$$

Substituting (2.7) into (2.6), we obtain (2.2). This completes the proof of the lemma.

Remark 2.1 According to Lemma 2.3, we get $u(t) \geq 0$, if $h(t) \geq 0, t \in J$. By (2.2), we have

$$
\begin{aligned}
& u(t)=-\int_{0}^{t} \frac{(t-s)^{\alpha-1}}{\Gamma(\alpha)} h(s) d s+M(t)+\frac{(n-1) t^{n-2}}{n-1-\mu} \int_{0}^{1} \frac{(1-s)^{\alpha-1}}{\Gamma(\alpha)} h(s) d s \\
& -\frac{\mu(n-1) t^{n-2}}{n-1-\mu} \int_{0}^{1} \frac{(1-s)^{\alpha}}{\alpha \Gamma(\alpha)} h(s) d s+\frac{(\mu \mathcal{M}-M(1))(n-1) t^{n-2}}{n-1-\mu} \\
& =-\int_{0}^{t} \frac{(t-s)^{\alpha-1}}{\Gamma(\alpha)} h(s) d s+\frac{(n-1) t^{n-2}}{\alpha(n-1-\mu)} \int_{0}^{1} \frac{\alpha(1-s)^{\alpha-1}-\mu(1-s)^{\alpha}}{\Gamma(\alpha)} h(s) d s \\
& +\frac{(n-1-\mu) M(t)+(\mu \mathcal{M}-M(1))(n-1) t^{n-2}}{n-1-\mu} \\
& =-\int_{0}^{t} \frac{(t-s)^{\alpha-1}}{\Gamma(\alpha)} h(s) d s+\frac{(n-1) t^{n-2}}{\alpha(n-1-\mu)} \int_{0}^{t} \frac{\alpha(1-s)^{\alpha-1}-\mu(1-s)^{\alpha}}{\Gamma(\alpha)} h(s) d s \\
& +\frac{(n-1) t^{n-2}}{\alpha(n-1-\mu)} \int_{t}^{1} \frac{\alpha(1-s)^{\alpha-1}-\mu(1-s)^{\alpha}}{\Gamma(\alpha)} h(s) d s \\
& +\frac{(n-1-\mu) M(t)+(\mu \mathcal{M}-M(1))(n-1) t^{n-2}}{n-1-\mu} \\
& =\frac{1}{\alpha(n-1-\mu)} \\
& \cdot \int_{0}^{t} \frac{(n-1) t^{n-2}\left(\alpha(1-s)^{\alpha-1}-\mu(1-s)^{\alpha}\right)-\alpha(n-1-\mu)(t-s)^{\alpha-1}}{\Gamma(\alpha)} h(s) d s \\
& +\frac{(n-1) t^{n-2}}{\alpha(n-1-\mu)} \int_{t}^{1} \frac{\alpha(1-s)^{\alpha-1}-\mu(1-s)^{\alpha}}{\Gamma(\alpha)} h(s) d s \\
& +\frac{(n-1-\mu) M(t)+(\mu \mathcal{M}-M(1))(n-1) t^{n-2}}{n-1-\mu} \text {. }
\end{aligned}
$$


Since $0 \leq \mu<n-1, n-1<\alpha \leq n$, for $0 \leq s \leq t \leq 1$, we have

$$
\begin{aligned}
& t^{n-2}(1-s)^{\alpha-1} \geq t^{\alpha-1}(1-s)^{\alpha-1}=(t-t s)^{\alpha-1} \geq(t-s)^{\alpha-1}, \\
& (n-1) t^{n-2}\left(\alpha(1-s)^{\alpha-1}-\mu(1-s)^{\alpha}\right)-\alpha(n-1-\mu)(t-s)^{\alpha-1} \\
& \quad=t^{n-2}(1-s)^{\alpha-1}((n-1) \alpha-\mu(1-s))-\alpha(n-1-\mu)(t-s)^{\alpha-1} \\
& \quad \geq(t-s)^{\alpha-1}((n-1) \alpha-\mu(1-s)-\alpha(n-1-\mu)) \\
& \quad=(t-s)^{\alpha-1}(\alpha-(1-s)) \mu \geq 0 .
\end{aligned}
$$

Thus, by (2.8) and (2.9), we know

$$
\begin{aligned}
& \frac{1}{\alpha(n-1-\mu)} \\
& \quad \int_{0}^{t} \frac{(n-1) t^{n-2}\left(\alpha(1-s)^{\alpha-1}-\mu(1-s)^{\alpha}\right)-\alpha(n-1-\mu)(t-s)^{\alpha-1}}{\Gamma(\alpha)} h(s) d s \geq 0 .
\end{aligned}
$$

For $0 \leq t \leq s \leq 1$, we have

$$
\alpha(1-s)^{\alpha-1}-\mu(1-s)^{\alpha}=(1-s)^{\alpha-1}(\alpha-\mu(1-s)) \geq 0 .
$$

Then we obtain

$$
\frac{(n-1) t^{n-2}}{\alpha(n-1-\mu)} \int_{t}^{1} \frac{\alpha(1-s)^{\alpha-1}-\mu(1-s)^{\alpha}}{\Gamma(\alpha)} h(s) d s \geq 0 .
$$

By the definition of $M(t), M(1)$ and $\mathcal{M}$, since $b_{i} \geq 0(i=1,2, \ldots, n-3, n-1)$, we know $M(t) \geq 0, M(1) \geq 0$ and $\mathcal{M} \geq 0$, so

$$
\begin{aligned}
& (n-1-\mu) M(t)+(\mu \mathcal{M}-M(1))(n-1) t^{n-2} \\
& \quad \geq(n-1-\mu)\left(M(t)+(\mu \mathcal{M}-M(1)) t^{n-2}\right) \\
& \quad=(n-1-\mu)\left(M(t)-M(1) t^{n-2}+\mu \mathcal{M} t^{n-2}\right) \geq 0 .
\end{aligned}
$$

From (2.10), (2.12), and (2.13), we get $u(t) \geq 0$, if $h(t) \geq 0, t \in J$.

Remark 2.2 By Remark 2.1, combined with the boundary conditions $0 \leq \mu<n-1, n \geq 3$, $b_{i} \geq 0(i=1,2, \ldots, n-3, n-1)$, we also get $u(t)>0, t \in(0,1)$, if $h(t) \not \equiv 0$.

Lemma 2.4 (Krasnoselskii fixed point theorem [28]) Let $X$ be a closed convex and nonempty subset of the Banach space E. Let $A$ and $B$ be two operators such that

1. $A x+B y \in X$, whenever $x, y \in X$;

2. A is compact and continuous;

3. $B$ is a contraction mapping.

Then there exists $z \in X$ such that $z=A z+B z$.

\section{Main results}

In the following, we list some conditions to be used later: 
$\left(\mathrm{H}_{1}\right) f: J \times \mathbb{R}_{+} \times \mathbb{R}_{+} \times \mathbb{R}_{+} \rightarrow \mathbb{R}_{+}$is measurable with respect to $t$ on $J$.

$\left(\mathrm{H}_{2}\right)$ There exist a constant $\alpha_{1} \in(0, \alpha-n+1)$ and real-valued functions $m_{1}(t), m_{2}(t), m_{3}(t) \in$ $L^{\frac{1}{\alpha_{1}}}\left(J, \mathbb{R}_{+}\right)$such that

$$
\begin{aligned}
& |f(t, u, v, w)-f(t, \bar{u}, \bar{v}, \bar{w})| \\
& \quad \leq m_{1}(t)|u-\bar{u}|+m_{2}(t)|v-\bar{v}|+m_{3}(t)|w-\bar{w}|, \quad t \in J, u, v, w, \bar{u}, \bar{v}, \bar{w} \in \mathbb{R}_{+} .
\end{aligned}
$$

$\left(\mathrm{H}_{3}\right)$ There exist a constant $\alpha_{2} \in(0, \alpha-n+1)$ and a real-valued function $h(t) \in L^{\frac{1}{\alpha_{2}}}\left(J, \mathbb{R}_{+}\right)$ such that

$$
|f(t, u, v, w)| \leq h(t), \quad t \in J, u, v, w \in D, D \subset \mathbb{R}_{+} \text {is a bounded subinterval. }
$$

Theorem 3.1 Assume that $\left(\mathrm{H}_{1}\right)-\left(\mathrm{H}_{3}\right)$ hold. If

$$
\begin{aligned}
\Omega= & \frac{\varpi}{\Gamma(\alpha)\left(\frac{\alpha-\alpha_{2}}{1-\alpha_{2}}\right)^{1-\alpha_{2}}}+\frac{(n-1)}{\Gamma(\alpha)(n-1-\mu)} \frac{\varpi}{\left(\frac{\alpha-\alpha_{2}}{1-\alpha_{2}}\right)^{1-\alpha_{2}}} \\
& +\frac{\mu(n-1)}{\alpha \Gamma(\alpha)(n-1-\mu)} \frac{\varpi}{\left(\frac{\alpha-\alpha_{2}+1}{1-\alpha_{2}}\right)^{1-\alpha_{2}}}<1,
\end{aligned}
$$

where $\varpi=\left\|m_{1}+k^{*} m_{2}+h^{*} m_{3}\right\|_{L^{\frac{1}{\alpha_{2}}}}$, then FBVP (1.1) has a unique positive solution on J.

Proof For $t \in J, u \in C\left(J, \mathbb{R}_{+}\right)$, by $\left(\mathrm{H}_{3}\right)$, we have

$$
\begin{aligned}
\int_{0}^{t}\left|(t-s)^{\alpha-1} f(s, u(s), T u(s), S u(s))\right| d s & \leq\left(\int_{0}^{t}(t-s)^{\frac{\alpha-1}{1-\alpha_{2}}} d s\right)^{1-\alpha_{2}}\left(\int_{0}^{t}(h(s))^{\frac{1}{\alpha_{2}}} d s\right)^{\alpha_{2}} \\
& \leq \frac{\|h\|_{L^{\frac{1}{\alpha_{2}}}}}{\left(\frac{\alpha-\alpha_{2}}{1-\alpha_{2}}\right)^{1-\alpha_{2}}} .
\end{aligned}
$$

Thus, $\left|(t-s)^{\alpha-1} f(s, u(s), T u(s), S u(s))\right|$ is Lebesgue integrable with respect to $s \in[0, t]$ for all $t \in J$ and $u \in C\left(J, \mathbb{R}_{+}\right)$. Hence, FBVP (1.1) is equivalent to the following integral equation:

$$
\begin{aligned}
u(t)= & -\int_{0}^{t} \frac{(t-s)^{\alpha-1}}{\Gamma(\alpha)} f(s, u(s), T u(s), S u(s)) d s \\
& +\frac{(n-1) t^{n-2}}{n-1-\mu} \int_{0}^{1} \frac{(1-s)^{\alpha-1}}{\Gamma(\alpha)} f(s, u(s), T u(s), S u(s)) d s \\
& -\frac{\mu(n-1) t^{n-2}}{n-1-\mu} \int_{0}^{1} \frac{(1-s)^{\alpha}}{\alpha \Gamma(\alpha)} f(s, u(s), T u(s), S u(s)) d s \\
& +M(t)+\frac{(\mu \mathcal{M}-M(1))(n-1) t^{n-2}}{n-1-\mu}, \quad t \in J .
\end{aligned}
$$

Let

$$
\begin{aligned}
r \geq & \frac{\|h\|_{L^{\frac{1}{\alpha_{2}}}}}{\Gamma(\alpha)\left(\frac{\alpha-\alpha_{2}}{1-\alpha_{2}}\right)^{1-\alpha_{2}}}+\frac{(n-1)}{\Gamma(\alpha)(n-1-\mu)} \frac{\|h\|_{L^{\frac{1}{\alpha_{2}}}}}{\left(\frac{\alpha-\alpha_{2}}{1-\alpha_{2}}\right)^{1-\alpha_{2}}} \\
& +\frac{\mu(n-1)}{\alpha \Gamma(\alpha)(n-1-\mu)} \frac{\|h\|_{L^{\frac{1}{\alpha_{2}}}}}{\left(\frac{\alpha-\alpha_{2}+1}{1-\alpha_{2}}\right)^{1-\alpha_{2}}}+M(1)+\frac{(\mu \mathcal{M}-M(1))(n-1)}{n-1-\mu} .
\end{aligned}
$$


Now, define the operator $F$ on $B_{r}=\left\{u \in C\left(J, \mathbb{R}_{+}\right):\|u\| \leq r\right\}$ as follows:

$$
\begin{aligned}
F u(t)= & -\int_{0}^{t} \frac{(t-s)^{\alpha-1}}{\Gamma(\alpha)} f(s, u(s), T u(s), S u(s)) d s \\
& +\frac{(n-1) t^{n-2}}{n-1-\mu} \int_{0}^{1} \frac{(1-s)^{\alpha-1}}{\Gamma(\alpha)} f(s, u(s), T u(s), S u(s)) d s \\
& -\frac{\mu(n-1) t^{n-2}}{n-1-\mu} \int_{0}^{1} \frac{(1-s)^{\alpha}}{\alpha \Gamma(\alpha)} f(s, u(s), T u(s), S u(s)) d s \\
& +M(t)+\frac{(\mu \mathcal{M}-M(1))(n-1) t^{n-2}}{n-1-\mu}, \quad t \in J .
\end{aligned}
$$

Therefore, the existence of a solution of the FBVP (1.1) is equivalent to that of a fixed point on $B_{r}$ of the operator $F$. We shall use the Banach contraction principle to prove that $F$ has a fixed point. The proof is divided into two steps.

1. $F u \in B_{r}$, for every $u \in B_{r}$.

For every $u \in B_{r}$ and $\delta>0$, by $\left(\mathrm{H}_{3}\right)$ and the Hölder's inequality, we get

$$
\begin{aligned}
& |F u(t+\delta)-F u(t)| \\
& \leq \mid-\int_{0}^{t+\delta} \frac{(t+\delta-s)^{\alpha-1}}{\Gamma(\alpha)} f(s, u(s), T u(s), \operatorname{Su}(s)) d s \\
& +\int_{0}^{t} \frac{(t-s)^{\alpha-1}}{\Gamma(\alpha)} f(s, u(s), T u(s), S u(s)) d s \\
& +\frac{(n-1)\left|(t+\delta)^{n-2}-t^{n-2}\right|}{n-1-\mu} \int_{0}^{1} \frac{(1-s)^{\alpha-1}}{\Gamma(\alpha)} f(s, u(s), T u(s), \operatorname{Su}(s)) d s \\
& +\frac{\mu(n-1)\left|(t+\delta)^{n-2}-t^{n-2}\right|}{n-1-\mu} \int_{0}^{1} \frac{(1-s)^{\alpha}}{\alpha \Gamma(\alpha)} f(s, u(s), T u(s), S u(s)) d s \\
& +|M(t+\delta)-M(t)|+\frac{(\mu \mathcal{M}-M(1))(n-1)\left|(t+\delta)^{n-2}-t^{n-2}\right|}{n-1-\mu} \\
& \leq \int_{0}^{t} \frac{\left|(t+\delta-s)^{\alpha-1}-(t-s)^{\alpha-1}\right|}{\Gamma(\alpha)} h(s) d s+\int_{t}^{t+\delta} \frac{(t-s)^{\alpha-1}}{\Gamma(\alpha)} h(s) d s \\
& +\frac{(n-1)\left|(t+\delta)^{n-2}-t^{n-2}\right|}{n-1-\mu} \int_{0}^{1} \frac{(1-s)^{\alpha-1}}{\Gamma(\alpha)} h(s) d s \\
& +\frac{\mu(n-1)\left|(t+\delta)^{n-2}-t^{n-2}\right|}{n-1-\mu} \int_{0}^{1} \frac{(1-s)^{\alpha}}{\alpha \Gamma(\alpha)} h(s) d s \\
& +|M(t+\delta)-M(t)|+\frac{(\mu \mathcal{M}-M(1))(n-1)\left|(t+\delta)^{n-2}-t^{n-2}\right|}{n-1-\mu} \\
& \leq \frac{1}{\Gamma(\alpha)}\left(\left(\int_{0}^{t}(t+\delta-s)^{\frac{\alpha-1}{1-\alpha_{2}}} d s\right)^{1-\alpha_{2}}-\left(\int_{0}^{t}(t-s)^{\frac{\alpha-1}{1-\alpha_{2}}} d s\right)^{1-\alpha_{2}}\right)\left(\int_{0}^{1}(h(s))^{\frac{1}{\alpha_{2}}} d s\right)^{\alpha_{2}} \\
& +\frac{1}{\Gamma(\alpha)}\left(\int_{t}^{t+\delta}(t+\delta-s)^{\frac{\alpha-1}{1-\alpha_{2}}} d s\right)^{1-\alpha_{2}}\left(\int_{0}^{1}(h(s))^{\frac{1}{\alpha_{2}}} d s\right)^{\alpha_{2}} \\
& +\frac{(n-1)\left|(t+\delta)^{n-2}-t^{n-2}\right|}{\Gamma(\alpha)(n-1-\mu)}\left(\int_{0}^{1}(1-s)^{\frac{\alpha-1}{1-\alpha_{2}}} d s\right)^{1-\alpha_{2}}\left(\int_{0}^{1}(h(s))^{\frac{1}{\alpha_{2}}} d s\right)^{\alpha_{2}} \\
& +\frac{\mu(n-1)\left|(t+\delta)^{n-2}-t^{n-2}\right|}{\alpha \Gamma(\alpha)(n-1-\mu)}\left(\int_{0}^{1}(1-s)^{\frac{\alpha}{1-\alpha_{2}}} d s\right)^{1-\alpha_{2}}\left(\int_{0}^{1}(h(s))^{\frac{1}{\alpha_{2}}} d s\right)^{\alpha_{2}}
\end{aligned}
$$




$$
\begin{aligned}
& +|M(t+\delta)-M(t)|+\frac{(\mu \mathcal{M}-M(1))(n-1)\left|(t+\delta)^{n-2}-t^{n-2}\right|}{n-1-\mu} \\
& \leq \frac{\|h\|_{L^{\frac{1}{\alpha 2}}}}{\Gamma(\alpha)}\left(\left(\frac{(t+\delta)^{\frac{\alpha-\alpha_{2}}{1-\alpha_{2}}}-\delta^{\frac{\alpha-\alpha_{2}}{1-\alpha_{2}}}}{\frac{\alpha-\alpha_{2}}{1-\alpha_{2}}}\right)^{1-\alpha_{2}}-\left(\frac{t^{\frac{\alpha-\alpha_{2}}{1-\alpha_{2}}}}{\frac{\alpha-\alpha_{2}}{1-\alpha_{2}}}\right)^{1-\alpha_{2}}+\left(\frac{\delta^{\frac{\alpha-\alpha_{2}}{1-\alpha_{2}}}}{\frac{\alpha-\alpha_{2}}{1-\alpha_{2}}}\right)^{1-\alpha_{2}}\right) \\
& +\frac{\|h\|_{L^{\frac{1}{\alpha_{2}}}}(n-1)\left|(t+\delta)^{n-2}-t^{n-2}\right|}{\Gamma(\alpha)(n-1-\mu)}\left(\frac{1-\alpha_{2}}{\alpha-\alpha_{2}}\right)^{1-\alpha_{2}} \\
& +\frac{\|h\|_{L^{\frac{1}{\alpha_{2}}}} \mu(n-1)\left|(t+\delta)^{n-2}-t^{n-2}\right|}{\alpha \Gamma(\alpha)(n-1-\mu)}\left(\frac{1-\alpha_{2}}{\alpha-\alpha_{2}+1}\right)^{1-\alpha_{2}} \\
& +|M(t+\delta)-M(t)|+\frac{(\mu \mathcal{M}-M(1))(n-1)\left|(t+\delta)^{n-2}-t^{n-2}\right|}{n-1-\mu} .
\end{aligned}
$$

Thus, the right-hand side of the above inequality tends to zero as $\delta \rightarrow 0$. Therefore, $F$ is continuous on $J$.

Next, we prove $F u(t) \geq 0, t \in J$. For any $t \in J$,

$$
\begin{aligned}
& F u(t)=-\int_{0}^{t} \frac{(t-s)^{\alpha-1}}{\Gamma(\alpha)} f(s, u(s), T u(s), S u(s)) d s \\
& +\frac{(n-1) t^{n-2}}{n-1-\mu} \int_{0}^{1} \frac{(1-s)^{\alpha-1}}{\Gamma(\alpha)} f(s, u(s), T u(s), \operatorname{Su}(s)) d s \\
& -\frac{\mu(n-1) t^{n-2}}{n-1-\mu} \int_{0}^{1} \frac{(1-s)^{\alpha}}{\alpha \Gamma(\alpha)} f(s, u(s), T u(s), S u(s)) d s \\
& +M(t)+\frac{(\mu \mathcal{M}-M(1))(n-1) t^{n-2}}{n-1-\mu} \\
& =-\int_{0}^{t} \frac{(t-s)^{\alpha-1}}{\Gamma(\alpha)} f(s, u(s), T u(s), S u(s)) d s \\
& +\frac{(n-1) t^{n-2}}{\alpha(n-1-\mu)} \int_{0}^{1} \frac{\alpha(1-s)^{\alpha-1}-\mu(1-s)^{\alpha}}{\Gamma(\alpha)} f(s, u(s), T u(s), S u(s)) d s \\
& +\frac{(n-1-\mu) M(t)+(\mu \mathcal{M}-M(1))(n-1) t^{n-2}}{n-1-\mu} \\
& =-\int_{0}^{t} \frac{(t-s)^{\alpha-1}}{\Gamma(\alpha)} f(s, u(s), T u(s), S u(s)) d s \\
& +\frac{(n-1) t^{n-2}}{\alpha(n-1-\mu)} \int_{0}^{t} \frac{\alpha(1-s)^{\alpha-1}-\mu(1-s)^{\alpha}}{\Gamma(\alpha)} f(s, u(s), T u(s), S u(s)) d s \\
& +\frac{(n-1) t^{n-2}}{\alpha(n-1-\mu)} \int_{t}^{1} \frac{\alpha(1-s)^{\alpha-1}-\mu(1-s)^{\alpha}}{\Gamma(\alpha)} f(s, u(s), T u(s), S u(s)) d s \\
& +\frac{(n-1-\mu) M(t)+(\mu \mathcal{M}-M(1))(n-1) t^{n-2}}{n-1-\mu} \\
& =\frac{1}{\alpha(n-1-\mu)} \int_{0}^{t} \frac{(n-1) t^{n-2}\left(\alpha(1-s)^{\alpha-1}-\mu(1-s)^{\alpha}\right)-\alpha(n-1-\mu)(t-s)^{\alpha-1}}{\Gamma(\alpha)} \\
& \text { · } f(s, u(s), T u(s), \operatorname{Su}(s)) d s \\
& +\frac{(n-1) t^{n-2}}{\alpha(n-1-\mu)} \int_{t}^{1} \frac{\alpha(1-s)^{\alpha-1}-\mu(1-s)^{\alpha}}{\Gamma(\alpha)} f(s, u(s), T u(s), S u(s)) d s \\
& +\frac{(n-1-\mu) M(t)+(\mu \mathcal{M}-M(1))(n-1) t^{n-2}}{n-1-\mu} \text {. }
\end{aligned}
$$


Since $0 \leq \mu<n-1, n-1<\alpha \leq n$, for $0 \leq s \leq t \leq 1$, by (2.8) and (2.9), we have

$$
\begin{aligned}
& \frac{1}{\alpha(n-1-\mu)} \int_{0}^{t} \frac{(n-1) t^{n-2}\left(\alpha(1-s)^{\alpha-1}-\mu(1-s)^{\alpha}\right)-\alpha(n-1-\mu)(t-s)^{\alpha-1}}{\Gamma(\alpha)} \\
& \cdot f(s, u(s), \operatorname{Tu}(s), \operatorname{Su}(s)) d s \geq 0 .
\end{aligned}
$$

For $0 \leq t \leq s \leq 1$, by (2.11), we know

$$
\frac{(n-1) t^{n-2}}{\alpha(n-1-\mu)} \int_{t}^{1} \frac{\alpha(1-s)^{\alpha-1}-\mu(1-s)^{\alpha}}{\Gamma(\alpha)} f(s, u(s), T u(s), S u(s)) d s \geq 0
$$

By the definition of $M(t), M(1)$, and $\mathcal{M}$, since $b_{i} \geq 0(i=1,2, \ldots, n-3, n-1)$, we know $M(t) \geq 0, M(1) \geq 0$ and $\mathcal{M} \geq 0$, thus

$$
\begin{aligned}
& (n-1-\mu) M(t)+(\mu \mathcal{M}-M(1))(n-1) t^{n-2} \\
& \quad \geq(n-1-\mu)\left(M(t)+(\mu \mathcal{M}-M(1)) t^{n-2}\right) \\
& \quad=(n-1-\mu)\left(M(t)-M(1) t^{n-2}+\mu \mathcal{M} t^{n-2}\right) \geq 0 .
\end{aligned}
$$

From (3.2)-(3.5), we obtain $F u(t) \geq 0, t \in J$. So, $F u \in C\left(J, \mathbb{R}_{+}\right)$.

Moreover, for any $u \in B_{r}$ and all $t \in J$, we have

$$
\begin{aligned}
& \|F u\| \leq \int_{0}^{t} \frac{(t-s)^{\alpha-1}}{\Gamma(\alpha)}|f(s, u(s), T u(s), S u(s))| d s \\
& +\frac{(n-1) t^{n-2}}{n-1-\mu} \int_{0}^{1} \frac{(1-s)^{\alpha-1}}{\Gamma(\alpha)}|f(s, u(s), T u(s), S u(s))| d s \\
& +\frac{\mu(n-1) t^{n-2}}{n-1-\mu} \int_{0}^{1} \frac{(1-s)^{\alpha}}{\alpha \Gamma(\alpha)}|f(s, u(s), T u(s), S u(s))| d s \\
& +M(t)+\frac{(\mu \mathcal{M}-M(1))(n-1) t^{n-2}}{n-1-\mu} \\
& \leq \int_{0}^{t} \frac{(t-s)^{\alpha-1}}{\Gamma(\alpha)} h(s) d s+\frac{(n-1) t^{n-2}}{n-1-\mu} \int_{0}^{1} \frac{(1-s)^{\alpha-1}}{\Gamma(\alpha)} h(s) d s \\
& +\frac{\mu(n-1) t^{n-2}}{n-1-\mu} \int_{0}^{1} \frac{(1-s)^{\alpha}}{\alpha \Gamma(\alpha)} h(s) d s+M(1)+\frac{(\mu \mathcal{M}-M(1))(n-1)}{n-1-\mu} \\
& \leq \frac{1}{\Gamma(\alpha)}\left(\int_{0}^{t}(t-s)^{\frac{\alpha-1}{1-\alpha_{2}}} d s\right)^{1-\alpha_{2}}\left(\int_{0}^{t}(h(s))^{\frac{1}{\alpha_{2}}} d s\right)^{\alpha_{2}} \\
& +\frac{(n-1)}{\Gamma(\alpha)(n-1-\mu)}\left(\int_{0}^{1}(1-s)^{\frac{\alpha-1}{1-\alpha_{2}}} d s\right)^{1-\alpha_{2}}\left(\int_{0}^{1}(h(s))^{\frac{1}{\alpha_{2}}} d s\right)^{\alpha_{2}} \\
& +\frac{\mu(n-1)}{\alpha \Gamma(\alpha)(n-1-\mu)}\left(\int_{0}^{1}(1-s)^{\frac{\alpha}{1-\alpha_{2}}} d s\right)^{1-\alpha_{2}}\left(\int_{0}^{1}(h(s))^{\frac{1}{\alpha_{2}}} d s\right)^{\alpha_{2}} \\
& +M(1)+\frac{(\mu \mathcal{M}-M(1))(n-1)}{n-1-\mu} \\
& \leq \frac{\|h\|_{L^{\frac{1}{\alpha_{2}}}}}{\Gamma(\alpha)\left(\frac{\alpha-\alpha_{2}}{1-\alpha_{2}}\right)^{1-\alpha_{2}}}+\frac{(n-1)}{\Gamma(\alpha)(n-1-\mu)} \frac{\|h\|_{L^{\frac{1}{\alpha_{2}}}}}{\left(\frac{\alpha-\alpha_{2}}{1-\alpha_{2}}\right)^{1-\alpha_{2}}}
\end{aligned}
$$




$$
\begin{aligned}
& +\frac{\mu(n-1)}{\alpha \Gamma(\alpha)(n-1-\mu)} \frac{\|h\|_{L^{\frac{1}{\alpha_{2}}}}}{\left(\frac{\alpha-\alpha_{2}+1}{1-\alpha_{2}}\right)^{1-\alpha_{2}}} \\
& +M(1)+\frac{(\mu \mathcal{M}-M(1))(n-1)}{n-1-\mu} \leq r
\end{aligned}
$$

which implies that $\|F u\| \leq r$. Thus, we can conclude that, for all $u \in B_{r}, F u \in B_{r}$, that is, $F: B_{r} \rightarrow B_{r}$.

2. $F$ is a contraction mapping on $B_{r}$.

For $u_{1}, u_{2} \in B_{r}$ and any $t \in J$, using $\left(\mathrm{H}_{2}\right)$ and the Hölder's inequality, we have

$$
\begin{aligned}
& \left|F u_{1}(t)-F u_{2}(t)\right| \\
& \leq \int_{0}^{t} \frac{(t-s)^{\alpha-1}}{\Gamma(\alpha)}\left|f\left(s, u_{1}(s), T u_{1}(s), S u_{1}(s)\right)-f\left(s, u_{2}(s), T u_{2}(s), S u_{2}(s)\right)\right| d s \\
& +\frac{(n-1) t^{n-2}}{n-1-\mu} \int_{0}^{1} \frac{(1-s)^{\alpha-1}}{\Gamma(\alpha)} \mid f\left(s, u_{1}(s), T u_{1}(s), S u_{1}(s)\right) \\
& -f\left(s, u_{2}(s), T u_{2}(s), S u_{2}(s)\right) \mid d s \\
& +\frac{\mu(n-1) t^{n-2}}{n-1-\mu} \int_{0}^{1} \frac{(1-s)^{\alpha}}{\alpha \Gamma(\alpha)} \mid f\left(s, u_{1}(s), T u_{1}(s), S u_{1}(s)\right) \\
& -f\left(s, u_{2}(s), T u_{2}(s), S u_{2}(s)\right) \mid d s \\
& \leq \int_{0}^{t} \frac{(t-s)^{\alpha-1}}{\Gamma(\alpha)}\left(m_{1}(s)\left|u_{1}(s)-u_{2}(s)\right|+m_{2}(s)\left|T u_{1}(s)-T u_{2}(s)\right|\right. \\
& \left.+m_{3}(s)\left|S u_{1}(s)-S u_{2}(s)\right|\right) d s \\
& +\frac{(n-1)}{n-1-\mu} \int_{0}^{1} \frac{(1-s)^{\alpha-1}}{\Gamma(\alpha)}\left(m_{1}(s)\left|u_{1}(s)-u_{2}(s)\right|+m_{2}(s)\left|T u_{1}(s)-T u_{2}(s)\right|\right. \\
& \left.+m_{3}(s)\left|S u_{1}(s)-S u_{2}(s)\right|\right) d s \\
& +\frac{\mu(n-1)}{n-1-\mu} \int_{0}^{1} \frac{(1-s)^{\alpha}}{\alpha \Gamma(\alpha)}\left(m_{1}(s)\left|u_{1}(s)-u_{2}(s)\right|+m_{2}(s)\left|T u_{1}(s)-T u_{2}(s)\right|\right. \\
& \left.+m_{3}(s)\left|S u_{1}(s)-S u_{2}(s)\right|\right) d s \\
& \leq \frac{\left\|u_{1}-u_{2}\right\|}{\Gamma(\alpha)} \int_{0}^{t}(t-s)^{\alpha-1}\left(m_{1}(s)+k^{*} m_{2}(s)+h^{*} m_{3}(s)\right) d s \\
& +\frac{\left\|u_{1}-u_{2}\right\|}{\Gamma(\alpha)} \frac{(n-1)}{n-1-\mu} \int_{0}^{1}(1-s)^{\alpha-1}\left(m_{1}(s)+k^{*} m_{2}(s)+h^{*} m_{3}(s)\right) d s \\
& +\frac{\left\|u_{1}-u_{2}\right\|}{\alpha \Gamma(\alpha)} \frac{\mu(n-1)}{n-1-\mu} \int_{0}^{1}(1-s)^{\alpha}\left(m_{1}(s)+k^{*} m_{2}(s)+h^{*} m_{3}(s)\right) d s \\
& \leq \frac{\left\|u_{1}-u_{2}\right\|}{\Gamma(\alpha)}\left(\int_{0}^{t}(t-s)^{\frac{\alpha-1}{1-\alpha_{2}}} d s\right)^{1-\alpha_{2}}\left(\int_{0}^{t}\left(m_{1}(s)+k^{*} m_{2}(s)+h^{*} m_{3}(s)\right)^{\frac{1}{\alpha_{2}}} d s\right)^{\alpha_{2}} \\
& +\frac{\left\|u_{1}-u_{2}\right\|(n-1)}{\Gamma(\alpha)(n-1-\mu)}\left(\int_{0}^{1}(1-s)^{\frac{\alpha-1}{1-\alpha_{2}}} d s\right)^{1-\alpha_{2}} \\
& \cdot\left(\int_{0}^{1}\left(m_{1}(s)+k^{*} m_{2}(s)+h^{*} m_{3}(s)\right)^{\frac{1}{\alpha_{2}}} d s\right)^{\alpha_{2}} \\
& +\frac{\left\|u_{1}-u_{2}\right\| \mu(n-1)}{\alpha \Gamma(\alpha)(n-1-\mu)}\left(\int_{0}^{1}(1-s)^{\frac{\alpha}{1-\alpha_{2}}} d s\right)^{1-\alpha_{2}}
\end{aligned}
$$




$$
\begin{gathered}
\cdot\left(\int_{0}^{1}\left(m_{1}(s)+k^{*} m_{2}(s)+h^{*} m_{3}(s)\right)^{\frac{1}{\alpha_{2}}} d s\right)^{\alpha_{2}} \\
\leq\left(\frac{\varpi}{\Gamma(\alpha)\left(\frac{\alpha-\alpha_{2}}{1-\alpha_{2}}\right)^{1-\alpha_{2}}}+\frac{(n-1)}{\Gamma(\alpha)(n-1-\mu)} \frac{\varpi}{\left(\frac{\alpha-\alpha_{2}}{1-\alpha_{2}}\right)^{1-\alpha_{2}}}\right. \\
\left.+\frac{\mu(n-1)}{\alpha \Gamma(\alpha)(n-1-\mu)} \frac{\varpi}{\left(\frac{\alpha-\alpha_{2}+1}{1-\alpha_{2}}\right)^{1-\alpha_{2}}}\right)\left\|u_{1}-u_{2}\right\| .
\end{gathered}
$$

So, we obtain

$$
\left\|F u_{1}-F u_{2}\right\| \leq \Omega\left\|u_{1}-u_{2}\right\|
$$

Thus, $F$ is a contraction mapping by the condition (3.1). By the Banach contraction principle, we can deduce that $F$ has an unique fixed point which is the unique positive solution of the FBVP (1.1).

Theorem 3.2 Assume that $f: J \times \mathbb{R}_{+} \times \mathbb{R}_{+} \times \mathbb{R}_{+} \rightarrow \mathbb{R}_{+}$is a continuous function, and $\left(H_{2}\right)$, $\left(H_{3}\right)$ hold. If

$$
\varpi^{*}<1, \quad \Omega^{*}=\frac{(n-1)}{\Gamma(\alpha)(n-1-\mu)} \frac{\varpi}{\left(\frac{\alpha-\alpha_{2}}{1-\alpha_{2}}\right)^{1-\alpha_{2}}}+\frac{\mu(n-1)}{\alpha \Gamma(\alpha)(n-1-\mu)} \frac{\varpi}{\left(\frac{\alpha-\alpha_{2}+1}{1-\alpha_{2}}\right)^{1-\alpha_{2}}}<1
$$

where $\varpi^{*}=\int_{0}^{1} \frac{(1-s)^{\alpha-1}}{\Gamma(\alpha)}\left(m_{1}(s)+k^{*} m_{2}(s)+h^{*} m_{3}(s)\right) d s, \varpi=\left\|m_{1}+k^{*} m_{2}+h^{*} m_{3}\right\|_{L^{\frac{1}{\alpha_{2}}}}$, then FBVP (1.1) has at least one positive solution on $J$.

Proof Choose

$$
\begin{aligned}
R \geq & (1-\varpi)^{-1}\left\{\frac{\Psi}{\Gamma(\alpha+1)}+\frac{(n-1)}{\Gamma(\alpha)(n-1-\mu)} \frac{\|h\|_{L^{\frac{1}{\alpha_{2}}}}}{\left(\frac{\alpha-\alpha_{2}}{1-\alpha_{2}}\right)^{1-\alpha_{2}}}\right. \\
& +\frac{\mu(n-1)}{\alpha \Gamma(\alpha)(n-1-\mu)} \frac{\|h\|_{L^{\frac{1}{\alpha_{2}}}}}{\left(\frac{\alpha-\alpha_{2}+1}{1-\alpha_{2}}\right)^{1-\alpha_{2}}} \\
& \left.+M(1)+\frac{(\mu \mathcal{M}-M(1))(n-1)}{n-1-\mu}\right\},
\end{aligned}
$$

where $\Psi=\max \{f(t, 0,0,0): t \in J\}$. Consider the set $B_{R}=\left\{u \in C\left(J, \mathbb{R}_{+}\right):\|u\| \leq R\right\}$, then $B_{R}$ is a closed, bounded and convex set of $C\left(J, \mathbb{R}_{+}\right)$. We define the operators $A$ and $B$ on $B_{R}$ as

$$
\begin{aligned}
A u(t)= & -\int_{0}^{t} \frac{(t-s)^{\alpha-1}}{\Gamma(\alpha)} f(s, u(s), T u(s), S u(s)) d s, \quad t \in J, \\
B u(t)= & \frac{(n-1) t^{n-2}}{n-1-\mu} \int_{0}^{1} \frac{(1-s)^{\alpha-1}}{\Gamma(\alpha)} f(s, u(s), T u(s), S u(s)) d s \\
& -\frac{\mu(n-1) t^{n-2}}{n-1-\mu} \int_{0}^{1} \frac{(1-s)^{\alpha}}{\alpha \Gamma(\alpha)} f(s, u(s), T u(s), S u(s)) d s+M(t) \\
& +\frac{(\mu \mathcal{M}-M(1))(n-1) t^{n-2}}{n-1-\mu}, \quad t \in J .
\end{aligned}
$$


By a similar proof to (3.2)-(3.5) in Theorem 3.1, we know $A u(t)+B u(t) \geq 0, t \in J$. For any $u \in B_{R}$, by $\left(\mathrm{H}_{2}\right)$ and the triangle inequality, we get

$$
\begin{aligned}
|f(t, u(t), T u(t), S u(t))| & \leq|f(t, u(t), T u(t), S u(t))-f(t, 0,0,0)|+|f(t, 0,0,0)| \\
& \leq m_{1}(t)|u(t)|+m_{2}(t)|T u(t)|+m_{3}(t)|S u(t)|+\Psi, \quad t \in J .
\end{aligned}
$$

Then, for any $u \in B_{R}$ and all $t \in J$, we have

$$
\begin{aligned}
|A u(t)| & =\left|-\int_{0}^{t} \frac{(t-s)^{\alpha-1}}{\Gamma(\alpha)} f(s, u(s), T u(s), S u(s)) d s\right| \\
& \leq \int_{0}^{t} \frac{(t-s)^{\alpha-1}}{\Gamma(\alpha)}\left(m_{1}(s)|u(s)|+m_{2}(s)|T u(s)|+m_{3}(s)|\operatorname{Su}(s)|+\Psi\right) d s \\
& \leq R \int_{0}^{1} \frac{(t-s)^{\alpha-1}}{\Gamma(\alpha)}\left(m_{1}(s)+k^{*} m_{2}(s)+h^{*} m_{3}(s)\right) d s+\Psi \int_{0}^{1} \frac{(t-s)^{\alpha-1}}{\Gamma(\alpha)} d s \\
& \leq \varpi^{*} R+\frac{\Psi}{\Gamma(\alpha+1)} .
\end{aligned}
$$

For any $v \in B_{R}$ and all $t \in J$, by $\left(\mathrm{H}_{2}\right)$, we have

$$
\begin{aligned}
& |B v(t)| \leq \frac{(n-1) t^{n-2}}{n-1-\mu} \int_{0}^{1} \frac{(1-s)^{\alpha-1}}{\Gamma(\alpha)}|f(s, v(s), T v(s), S v(s))| d s \\
& +\frac{\mu(n-1) t^{n-2}}{n-1-\mu} \int_{0}^{1} \frac{(1-s)^{\alpha}}{\alpha \Gamma(\alpha)}|f(s, v(s), T \nu(s), S v(s))| d s \\
& +M(t)+\frac{(\mu \mathcal{M}-M(1))(n-1) t^{n-2}}{n-1-\mu} \\
& \leq \frac{(n-1) t^{n-2}}{n-1-\mu} \int_{0}^{1} \frac{(1-s)^{\alpha-1}}{\Gamma(\alpha)} h(s) d s+\frac{\mu(n-1) t^{n-2}}{n-1-\mu} \int_{0}^{1} \frac{(1-s)^{\alpha}}{\alpha \Gamma(\alpha)} h(s) d s \\
& +M(1)+\frac{(\mu \mathcal{M}-M(1))(n-1)}{n-1-\mu} \\
& \leq \frac{(n-1)}{\Gamma(\alpha)(n-1-\mu)}\left(\int_{0}^{1}(1-s)^{\frac{\alpha-1}{1-\alpha_{2}}} d s\right)^{1-\alpha_{2}}\left(\int_{0}^{1}(h(s))^{\frac{1}{\alpha_{2}}} d s\right)^{\alpha_{2}} \\
& +\frac{\mu(n-1)}{\alpha \Gamma(\alpha)(n-1-\mu)}\left(\int_{0}^{1}(1-s)^{\frac{\alpha}{1-\alpha_{2}}} d s\right)^{1-\alpha_{2}}\left(\int_{0}^{1}(h(s))^{\frac{1}{\alpha_{2}}} d s\right)^{\alpha_{2}} \\
& +M(1)+\frac{(\mu \mathcal{M}-M(1))(n-1)}{n-1-\mu} \\
& \leq \frac{(n-1)}{\Gamma(\alpha)(n-1-\mu)} \frac{\|h\|_{L^{\frac{1}{\alpha_{2}}}}}{\left(\frac{\alpha-\alpha_{2}}{1-\alpha_{2}}\right)^{1-\alpha_{2}}}+\frac{\mu(n-1)}{\alpha \Gamma(\alpha)(n-1-\mu)} \frac{\|h\|_{L^{\frac{1}{\alpha_{2}}}}}{\left(\frac{\alpha-\alpha_{2}+1}{1-\alpha_{2}}\right)^{1-\alpha_{2}}} \\
& +M(1)+\frac{(\mu \mathcal{M}-M(1))(n-1)}{n-1-\mu} \text {. }
\end{aligned}
$$

By (3.6)-(3.7), we obtain $|A u(t)+B v(t)| \leq|A u(t)|+|B u(t)| \leq R, u, v \in B_{R}, t \in J$.

For $v_{1}, v_{2} \in B_{R}$ and any $t \in J$, using $\left(\mathrm{H}_{2}\right)$ and the Hölder's inequality, we have

$$
\begin{aligned}
& \left|B v_{1}(t)-B v_{2}(t)\right| \\
& \quad \leq \frac{(n-1) t^{n-2}}{n-1-\mu} \int_{0}^{1} \frac{(1-s)^{\alpha-1}}{\Gamma(\alpha)}\left|f\left(s, v_{1}(s), T v_{1}(s), S v_{1}(s)\right)-f\left(s, v_{2}(s), T v_{2}(s), S v_{2}(s)\right)\right| d s
\end{aligned}
$$




$$
\begin{aligned}
& +\frac{\mu(n-1) t^{n-2}}{n-1-\mu} \int_{0}^{1} \frac{(1-s)^{\alpha}}{\alpha \Gamma(\alpha)}\left|f\left(s, v_{1}(s), T v_{1}(s), S v_{1}(s)\right)-f\left(s, v_{2}(s), T v_{2}(s), S v_{2}(s)\right)\right| d s \\
& \leq \frac{(n-1)}{n-1-\mu} \int_{0}^{1} \frac{(1-s)^{\alpha-1}}{\Gamma(\alpha)}\left(m_{1}(s)\left|v_{1}(s)-v_{2}(s)\right|+m_{2}(s)\left|T v_{1}(s)-T v_{2}(s)\right|\right. \\
& \left.+m_{3}(s)\left|S v_{1}(s)-S v_{2}(s)\right|\right) d s \\
& +\frac{\mu(n-1)}{n-1-\mu} \int_{0}^{1} \frac{(1-s)^{\alpha}}{\alpha \Gamma(\alpha)}\left(m_{1}(s)\left|v_{1}(s)-v_{2}(s)\right|+m_{2}(s)\left|T v_{1}(s)-T v_{2}(s)\right|\right. \\
& \left.+m_{3}(s)\left|S v_{1}(s)-S v_{2}(s)\right|\right) d s \\
& \leq \frac{\left\|v_{1}-v_{2}\right\|}{\Gamma(\alpha)} \frac{(n-1)}{n-1-\mu} \int_{0}^{1}(1-s)^{\alpha-1}\left(m_{1}(s)+k^{*} m_{2}(s)+h^{*} m_{3}(s)\right) d s \\
& +\frac{\left\|v_{1}-v_{2}\right\|}{\alpha \Gamma(\alpha)} \frac{\mu(n-1)}{n-1-\mu} \int_{0}^{1}(1-s)^{\alpha}\left(m_{1}(s)+k^{*} m_{2}(s)+h^{*} m_{3}(s)\right) d s \\
& \leq \frac{\left\|v_{1}-v_{2}\right\|}{\Gamma(\alpha)}\left(\int_{0}^{t}(t-s)^{\frac{\alpha-1}{1-\alpha_{2}}} d s\right)^{1-\alpha_{2}}\left(\int_{0}^{t}\left(m_{1}(s)+k^{*} m_{2}(s)+h^{*} m_{3}(s)\right)^{\frac{1}{\alpha_{2}}} d s\right)^{\alpha_{2}} \\
& +\frac{\left\|v_{1}-v_{2}\right\|(n-1)}{\Gamma(\alpha)(n-1-\mu)}\left(\int_{0}^{1}(1-s)^{\frac{\alpha-1}{1-\alpha_{2}}} d s\right)^{1-\alpha_{2}} \\
& \cdot\left(\int_{0}^{1}\left(m_{1}(s)+k^{*} m_{2}(s)+h^{*} m_{3}(s)\right)^{\frac{1}{\alpha_{2}}} d s\right)^{\alpha_{2}} \\
& +\frac{\left\|v_{1}-v_{2}\right\| \mu(n-1)}{\alpha \Gamma(\alpha)(n-1-\mu)}\left(\int_{0}^{1}(1-s)^{\frac{\alpha}{1-\alpha_{2}}} d s\right)^{1-\alpha_{2}} \\
& \cdot\left(\int_{0}^{1}\left(m_{1}(s)+k^{*} m_{2}(s)+h^{*} m_{3}(s)\right)^{\frac{1}{\alpha_{2}}} d s\right)^{\alpha_{2}} \\
& \leq\left(\frac{(n-1)}{\Gamma(\alpha)(n-1-\mu)} \frac{\varpi}{\left(\frac{\alpha-\alpha_{2}}{1-\alpha_{2}}\right)^{1-\alpha_{2}}}+\frac{\mu(n-1)}{\alpha \Gamma(\alpha)(n-1-\mu)} \frac{\varpi}{\left(\frac{\alpha-\alpha_{2}+1}{1-\alpha_{2}}\right)^{1-\alpha_{2}}}\right)\left\|v_{1}-v_{2}\right\| .
\end{aligned}
$$

Therefore, we get

$$
\left\|B v_{1}-B v_{2}\right\| \leq \Omega^{*}\left\|v_{1}-v_{2}\right\|
$$

It follows from $\left(\mathrm{H}_{2}\right)$ that $B$ is a contraction mapping for $\Omega^{*}<1$.

The continuity of $f$ implies that $A$ is continuous. Also, $A$ is uniformly bounded on $B_{R}$, since for any $u \in B_{R}$, by $\left(\mathrm{H}_{3}\right)$, we have

$$
\begin{aligned}
\|A u\| & \leq \int_{0}^{t} \frac{(t-s)^{\alpha-1}}{\Gamma(\alpha)}|f(s, u(s), T u(s), S u(s))| d s \leq \int_{0}^{t} \frac{(t-s)^{\alpha-1}}{\Gamma(\alpha)} h(s) d s \\
& \leq \frac{1}{\Gamma(\alpha)}\left(\int_{0}^{t}(t-s)^{\frac{\alpha-1}{1-\alpha_{2}}} d s\right)^{1-\alpha_{2}}\left(\int_{0}^{t}(h(s))^{\frac{1}{\alpha_{2}}} d s\right)^{\alpha_{2}} \\
& \leq \frac{\|h\|_{L^{\frac{1}{\alpha_{2}}}}}{\Gamma(\alpha)\left(\frac{\alpha-\alpha_{2}}{1-\alpha_{2}}\right)^{1-\alpha_{2}}} .
\end{aligned}
$$

On the other hand, for any $u \in B_{R}, t_{1}, t_{2} \in J$, without loss of generality, we may assume $t_{1}<t_{2}$, for any $\varepsilon>0$, choose $\delta=\frac{1}{2}\left(\frac{\varepsilon \Gamma(\alpha)}{\Phi}\right)^{\frac{1}{\alpha}}, \Phi=\sup \left\{f(t, u, v, w):(t, u, v, w) \in J \times B_{R} \times B_{k^{*} R} \times\right.$ $\left.B_{h^{*} R}\right\}, B_{k^{*} R}=\left\{v \in C\left(J, \mathbb{R}_{+}\right):\|v\| \leq k^{*} R\right\}, B_{h^{*} R}=\left\{w \in C\left(J, \mathbb{R}_{+}\right):\|w\| \leq h^{*} R\right\}$, such that, for 
$0<t_{2}-t_{1}<\delta$, we have

$$
\begin{aligned}
& \left|A u\left(t_{2}\right)-A u\left(t_{1}\right)\right| \\
& \quad \leq\left|\int_{0}^{t_{2}} \frac{\left(t_{2}-s\right)^{\alpha-1}}{\Gamma(\alpha)} f(s, u(s), T u(s), S u(s)) d s-\int_{0}^{t_{1}} \frac{\left(t_{1}-s\right)^{\alpha-1}}{\Gamma(\alpha)} f(s, u(s), T u(s), S u(s)) d s\right| \\
& \quad \leq \frac{\Phi}{\Gamma(\alpha)}\left|\int_{0}^{t_{1}}\left(\left(t_{2}-s\right)^{\alpha-1}-\left(t_{1}-s\right)^{\alpha-1}\right) d s+\int_{t_{1}}^{t_{2}}\left(t_{1}-s\right)^{\alpha-1} d s\right| \\
& \quad=\frac{\Phi}{\alpha \Gamma(\alpha)}\left|t_{2}^{\alpha}-t_{1}^{\alpha}\right| .
\end{aligned}
$$

Since $0<t_{2}-t_{1}<\delta$, we have the following two cases:

Case 1. $\delta \leq t_{1}<t_{2} \leq 1$.

$$
\left|A u\left(t_{2}\right)-A u\left(t_{1}\right)\right| \leq \frac{\Phi}{\alpha \Gamma(\alpha)}\left|t_{2}^{\alpha}-t_{1}^{\alpha}\right| \leq \frac{\Phi}{\alpha \Gamma(\alpha)} \alpha \delta^{\alpha-1}\left(t_{2}-t_{1}\right) \leq \frac{\Phi}{\Gamma(\alpha)} \delta^{\alpha}<\varepsilon .
$$

Case $2.0 \leq t_{1}<\delta, t_{2} \leq 2 \delta \leq 1$.

$$
\left|A u\left(t_{2}\right)-A u\left(t_{1}\right)\right| \leq \frac{\Phi}{\alpha \Gamma(\alpha)}\left|t_{2}^{\alpha}-t_{1}^{\alpha}\right| \leq \frac{\Phi}{\alpha \Gamma(\alpha)} t_{2}^{\alpha} \leq \frac{\Phi}{\alpha \Gamma(\alpha)}(2 \delta)^{\alpha}<\varepsilon .
$$

Therefore, $A$ is equicontinuous, by the Arzela-Ascoli theorem, we know that $A$ is compact on $B_{R}$, so the operator $A$ is completely continuous. Thus, all the assumptions of Lemma 2.4 are satisfied, by Lemma 2.4, FBVP (1.1) has at least one positive solution on $J$.

\section{Example}

Now we consider the existence and uniqueness of positive solutions for the fractional differential system:

$$
\left\{\begin{array}{l}
{ }^{c} D^{\alpha} u(t)+f(t, u(t), T u(t), S u(t))=0, \quad 0<t<1, \\
u(0)=1, \quad u^{\prime}(0)=2, \quad u^{(3)}(0)=0, \quad u(1)=\frac{\sin 1}{1-\cos 1} \int_{0}^{1} u(s) d s
\end{array}\right.
$$

where $3<\alpha<4,0<\mu=\frac{\sin 1}{1-\cos 1}<3$. Let $k>0$ be a constant, choose

$$
f(t, u, v, w)=\frac{e^{-t}}{1+k} \frac{|u|}{1+|u|}+\int_{0}^{t} \frac{e^{-(s-t)}}{1+k} \frac{|v|}{1+|v|} d s++\int_{0}^{1} \frac{e^{-t}(s+1)}{16\left(1+k^{2}\right)} \frac{|w|}{1+|w|} d s .
$$

So

$$
\begin{aligned}
& K(t, s)=\frac{e^{-(s-t)}}{1+k}, \quad H(t, s)=\frac{e^{-t}(s+1)}{16\left(1+k^{2}\right)}, \\
& k^{*}=\sup _{t \in J} \int_{0}^{t} K(t, s) d s=\frac{e-1}{(1+k)}, \quad h^{*}=\sup _{t \in J} \int_{0}^{1} H(t, s) d s=\frac{3}{32\left(1+k^{2}\right)}, \\
& |f(t, u, v, w)-f(t, \bar{u}, \bar{v}, \bar{w})| \\
& \quad \leq \frac{e^{-t}}{1+k}|u-\bar{u}|+\frac{e^{-k t}}{1+k}|v-\bar{v}|+\frac{e^{-t}}{8\left(1+k^{2}\right)}|w-\bar{w}|, \quad t \in J, u, v, w, \bar{u}, \bar{v}, \bar{w} \in \mathbb{R}_{+}, \\
& |f(t, u, v, w)| \leq \frac{e^{-t}+e^{-k t}}{1+k}+\frac{e^{-t}}{8\left(1+k^{2}\right)}, \quad t \in J, u, v, w \in \mathbb{R}_{+} .
\end{aligned}
$$


For $t \in J, \alpha_{1} \in(0, \alpha-3), \alpha_{2} \in(0, \alpha-3)$, set $m_{1}(t)=\frac{e^{-t}}{1+k}, m_{2}(t)=\frac{e^{-k t}}{1+k}, m_{3}(t)=\frac{e^{-t}}{8\left(1+k^{2}\right)} \in$ $L^{\frac{1}{\alpha_{1}}}\left(J, \mathbb{R}_{+}\right), h(t)=\frac{e^{-t}+e^{-k t}}{1+k}+\frac{e^{-t}}{8\left(1+k^{2}\right)} \in L^{\frac{1}{\alpha_{2}}}\left(J, \mathbb{R}_{+}\right), \varpi=\left\|\frac{e^{-t}}{1+k}+\frac{e^{-k t}(e-1)}{(1+k)^{2}}+\frac{3 e^{-t}}{256\left(1+k^{2}\right)^{2}}\right\|_{L^{\frac{1}{\alpha_{2}}}}$. Choosing some $k>0$ large enough and suitable $\alpha_{1} \in(0, \alpha-3), \alpha_{2} \in(0, \alpha-3)$, we can arrive at the inequality (3.1). Therefore, by Theorem 3.1, we see that FBVP (4.1) has a unique solution on $J$.

\section{Conclusions}

In this work, we establish the conditions of uniqueness and existence results of positive solutions for a class of fractional integro-differential equations involving the Caputo derivative of order $\alpha(n-1<\alpha \leq n, n \geq 3)$, the explicit cases, including measurable or continuous nonlinear $\operatorname{term} f$, are discussed by adopting new assumption conditions. Our results (Theorems 3.1 and 3.2) are based on the Banach contraction principle and the Krasnoselskii fixed point theorem. In particular, an example is given to show the effectiveness of the obtained results. Moreover, the sufficient conditions we obtained are very simple, which provides flexibility for the application and analysis of a nonlinear fractional differential equation.

\section{Competing interests}

The authors declare that they have no competing interests.

\section{Authors' contributions}

All authors contributed equally and significantly in writing this article. All authors read and approved the final manuscript.

\section{Author details}

'School of Science, Linyi University, Linyi, Shandong 276000, People's Republic of China. ${ }^{2}$ School of Mathematical Sciences, Qufu Normal University, Qufu, Shandong 273165, People's Republic of China. ${ }^{3}$ Department of Mathematics and Statistics, Curtin University, Perth, WA 6845, Australia.

\section{Acknowledgements}

The authors were supported financially by the National Natural Science Foundation of China (11626125, 11371221, 11571296), the Natural Science Foundation of Shandong Province of China (ZR2016AP04), a Project of Shandong Province Higher Educational Science and Technology Program (J16LI03, J14LI08), and the Applied Mathematics Enhancement Program of Linyi University and the Science Research Foundation for Doctoral Authorities of Linyi University (LYDX2016BS080).

Received: 12 May 2016 Accepted: 22 December 2016 Published online: 13 January 2017

\section{References}

1. Atanackovic, TM, Stankovic, B: On a class of differential equations with left and right fractional derivatives. Z. Angew. Math. Mech. 87, 537-546 (2009)

2. Debnath, L: Recent applications of fractional calculus to science and engineering. Int. J. Math. Math. Sci. 54 3413-3442 (2003)

3. $\mathrm{Xu}, \mathrm{H}$ : Analytical approximations for a population growth model with fractional order. Commun. Nonlinear Sci. Numer. Simul. 14, 1978-1983 (2009)

4. Kempfle, S, Schäfer, I, Beyer, H: Fractional calculus via functional calculus: theory and applications. Nonlinear Dyn. 29, 99-127 (2002)

5. Kilbas, AA, Srivastava, HM, Trujillo, JJ: Theory and Applications of Fractional Differential Equations. Elsevier, Amsterdam (2006)

6. Scudo, FM: Vito Volterra and theoretical ecology. Theor. Popul. Biol. 2, 1-23 (1971)

7. TeBeest, KG: Numerical and analytical solutions of Volterra's population model. SIAM Rev. 39, 484-493 (1997)

8. He, J: Nonlinear oscillation with fractional derivative and its applications. In: International Conference on Vibrating Engineering, Dalian, China (1998)

9. He, J: Some applications of nonlinear fractional differential equations and their approximations. Bull. Sci. Technol. 15, 86-90 (1999)

10. Bai, Z, Qiu, T: Existence of positive solution for singular fractional differential equation. Appl. Math. Comput. 215 2761-2767 (2009)

11. Li, H, Liu, L, Wu, Y: Positive solutions for singular nonlinear fractional differential equation with integral boundary conditions. Bound. Value Probl. 2015, 232 (2015)

12. Graef, JR, Kong, L: Existence of positive solutions to a higher order singular boundary value problem with fractional Q-derivatives. Fract. Calc. Appl. Anal. 16(3), 695-708 (2013)

13. Wang, Y, Liu, L, Wu, Y: Positive solutions for a class of higher-order singular semipositone fractional differential systems with coupled integral boundary conditions and parameters. Adv. Differ. Equ. 2014, 268 (2014) 
14. Cabada, A, Wang, G: Positive solutions of nonlinear fractional differential equations with integral boundary value conditions. J. Math. Anal. Appl. 389, 403-411 (2012)

15. Guo, L, Liu, L, Wu, Y: Existence of positive solutions for singular higher-order fractional differential equations with infinite-point boundary conditions. Bound. Value Probl. 2016, 114 (2016)

16. Zhang, $X, L i u, L, W u, Y:$ The uniqueness of positive solution for a fractional order model of turbulent flow in a porous medium. Appl. Math. Lett. 37, 26-33 (2014)

17. $\mathrm{Xu}, \mathrm{Y}, \mathrm{He}, \mathrm{Z}$ : Existence of solutions for nonlinear high-order fractional boundary value problem with integral boundary condition. J. Appl. Math. Comput. 44, 417-435 (2014)

18. Wang, Y, Liu, L, Zhang, X, Wu, Y: Positive solutions of an abstract fractional semipositone differential system model for bioprocesses of HIV infection. Appl. Math. Comput. 258, 312-324 (2015)

19. Wang, Y, Zhang, J: Positive solutions for higher-order singular fractional differential system with coupled integral boundary conditions. Adv. Differ. Equ. 2016, 117 (2016)

20. Liu, L, Zhang, X, Jiang, J, Wu, Y: The unique solution of a class of sum mixed monotone operator equations and its application to fractional boundary value problems. J. Nonlinear Sci. Appl. 9, 2943-2958 (2016)

21. Sun, F, Liu, L, Zhang, X, Wu, Y: Spectral analysis for a singular differential system with integral boundary conditions. Mediterr. J. Math. 13, 4763-4782 (2016)

22. Hao, X, Liu, L, Wu, Y: Positive solutions for nonlinear fractional semipositone differential equation with nonlocal boundary conditions. J. Nonlinear Sci. Appl. 9, 3992-4002 (2016)

23. $G u o, L, L i u, L, W u, Y:$ Uniqueness of iterative positive solutions for the singular fractional differential equations with integral boundary conditions. Bound. Value Probl. 2016, 147 (2016)

24. Guo, L, Liu, L, Wu, Y: Existence of positive solutions for singular fractional differential equations with infinite-point boundary conditions. Nonlinear Anal., Model. Control 21(5), 635-650 (2016)

25. Jiang, J, Liu, L: Existence of solutions for a sequential fractional differential system with coupled boundary conditions. Bound. Value Probl. 2016, 159 (2016)

26. Miller, KS, Ross, B: An Introduction to the Fractional Calculus and Fractional Differential Equations. Wiley, New York (1993)

27. Podlubny, l: Fractional Differential Equations. Mathematics in Science and Engineering, vol. 198. Academic Press, New York (1999)

28. Smart, DR: Fixed Point Theorems. Cambridge University Press, Cambridge (1980)

\section{Submit your manuscript to a SpringerOpen ${ }^{\ominus}$ journal and benefit from:}

- Convenient online submission

Rigorous peer review

- Immediate publication on acceptance

- Open access: articles freely available online

- High visibility within the field

- Retaining the copyright to your article 